\title{
Two cycloartanes-type triterpenoid from Homonoia riparia Lour
}

\author{
Le Thi Huyen ${ }^{1}$, Nguyen Thi Son ${ }^{1}$, Phan Van Kiem ${ }^{2 *}$ \\ ${ }^{1}$ University of Science, Vietnam National University (VNU) \\ ${ }^{2}$ Institute of Marine Biochemistry, Vietnam Academy of Science and Technology (VAST)
}

Received 3 June 2017; Accepted for publication 28 August 2017

\begin{abstract}
Using various chromatography methods, two known cycloartanes, 24-methylenecycloartane- $3 \beta, 6 \beta, 7 \beta, 16 \beta$-tetraol (1) and riparsaponin (2), together with two known flavonol glycosides, quercetin 3-O- $\beta$-D-glucopyranoside (3) and quercetin 3-O- $\alpha$ - L-rhamnopyranosyl $(1 \rightarrow 6) \beta$-D-glucopyranoside (4) were isolated from the methanol extract of the leaves of Homonoia riparia Lour. Their structures were determined by MS and 1D-, 2D-NMR spectra, as well as by comparison with those reported in the literature.
\end{abstract}

Keywords. Homonoia riparia, cycloartane, flavonol glycoside.

\section{INTRODUCTION}

Homonoia is a small genus of shrubs which belongs to the Euphorbiaceae family that is widespread from India to mainland China, Taiwan, and Vietnam, and found commonly at riverbanks [1]. The plant is used for the treatment of various diseases such as hepatitis, pneumonia, scald, and skin diseases $[1,2]$. The phytochemical studies of $H$. riparia confirmed the presence of sterols, triterpenes, and flavonoids [3, 4]. In this paper, we report herein the isolation and structure elucidation of two cycloartane-type triterpenoids and two flavonol glycosides from the methanol extract of the $H$. riparia leaves.

\section{MATERIAL AND METHODS}

\subsection{Plant materials}

The leaves of $H$. riparia collected in Vinhphuc, Vietnam, in March 2016, and identified by Prof. Ninh Khac Ban, Institute of Marine Biochemistry, Vietnam Academy of Science and Technology. A voucher specimen (HR01) was deposited at Lab of Pharmaceutical Chemistry, Faculty of Chemistry, University of Science, VNU.

\subsection{General experimental procedures}

The ${ }^{1} \mathrm{H}-\mathrm{NMR}(400 \mathrm{MHz})$ and ${ }^{13} \mathrm{C}-\mathrm{NMR}(100 \mathrm{MHz})$ spectra were recorded on a Variant AM400 FTNMR spectrometer and TMS was used as an internal standard. Column chromatography was performed using a silica gel (Kieselgel 60, 70-230 mesh and 230-400 mesh, Merck, Whitehouse Station, NJ) or RP-18 resins (30-50 $\mu \mathrm{m}$, Fuji silysia Chemical Ltd.), and thin layer chromatography (TLC) using precoated silica gel $60 \mathrm{~F}_{254}(0.25 \mathrm{~mm}$, Merck $)$ and $\mathrm{RP}-18 \mathrm{~F}_{254 \mathrm{~S}}$ plates $(0.25 \mathrm{~mm}$, Merck).

\subsection{Extraction and isolation}

The dried leaves of $H$. riparia $(5.0 \mathrm{~kg})$ were extracted with methanol $(8.0 \mathrm{~L})$ under sonication for $4 \mathrm{~h}$ to yield $700.0 \mathrm{~g}$ extract. This extract was suspended in $\mathrm{H}_{2} \mathrm{O}$ and successively partitioned with $n$-hexane and EtOAc to obtain the $n$-hexane (HR1, $19.8 \mathrm{~g}$ ), EtOAc (HR2, $253.5 \mathrm{~g}$ ), and aqueous (HR3, $430.0 \mathrm{~g})$ fractions. The fraction HR2 $(253.5 \mathrm{~g})$ was chromatographed on a silica gel column, eluting with a gradient solvent of $\mathrm{CHCl}_{3}-\mathrm{MeOH}(20: 1 \rightarrow 1$ : $1, \mathrm{v} / \mathrm{v})$ to obtain five sub-fractions, HR1A $(11.4 \mathrm{~g})$, HR1B (8.7 g), HR1C (3.5 g), HR1D (3.9 g), and HR1E $(55.8 \mathrm{~g})$. The fraction HR1D was chromatographed on a silica gel column eluting with $\mathrm{CHCl}_{3}: \mathrm{MeOH}(20: 1, \mathrm{v} / \mathrm{v})$ to give three smaller fractions, HR1C1 (1.7 g), HR1C2 (1.0 g), and HR1C3 $(0.8 \mathrm{~g})$. The fraction HR1C2 was chromatographed on HPLC using J'sphere ODS H$80(250 \mathrm{~mm}$ x $20 \mathrm{~mm}, 4 \mathrm{~mm}, 8 \mathrm{~nm})$ column eluting with $25 \%$ aqueous acetonitrile at a flow rate of 3.0 $\mathrm{mL} / \mathrm{min}$ to yield $\mathbf{1}(16.2 \mathrm{mg})$ and $\mathbf{2}(7.5 \mathrm{mg})$. The HR1E fraction was chromatographed on a silica gel column eluting with $\mathrm{CHCl}_{3}-\mathrm{MeOH}(6: 1, \mathrm{v} / \mathrm{v})$ to yield $\mathbf{3}(5.6 \mathrm{mg})$, and 4 (4.8 $\mathrm{mg})$. 


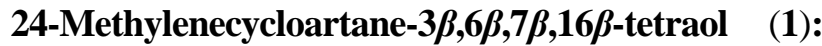
White amorphous powder; $[\alpha]_{D}^{25}:+42.5$ (c 0.1 , in $\mathrm{CD}_{3} \mathrm{OD}$ ); ESI-MS m/z: $511[\mathrm{M}+\mathrm{Na}]^{+} ; \mathrm{C}_{31} \mathrm{H}_{52} \mathrm{O}_{4}, \mathrm{M}=$ 488.38; ${ }^{1} \mathrm{H}$ - and ${ }^{13} \mathrm{C}-\mathrm{NMR}\left(\mathrm{CD}_{3} \mathrm{OD}\right)$, see table 1 .

Riparsaponin (2): White amorphous powder; $[\alpha]_{D}^{25}$ : +25.0 ( $c$ 0.1, in $\mathrm{CD}_{3} \mathrm{OD}$ ); ESI-MS $m / z: 643[\mathrm{M}+\mathrm{Na}]^{+}$; $\mathrm{C}_{36} \mathrm{H}_{60} \mathrm{O}_{8}, \mathrm{M}=620.43 ;{ }^{1} \mathrm{H}$ - and ${ }^{13} \mathrm{C}-\mathrm{NMR}$ (piridine$\mathrm{d}_{5}$ ): see Table 1 .

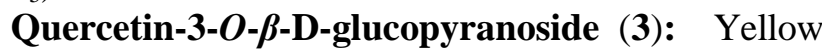
amorphous powder; ESI-MS $m / z: 465[\mathrm{M}+\mathrm{H}]^{+}$; $\mathrm{C}_{21} \mathrm{H}_{20} \mathrm{O}_{12}, \mathrm{M}=464.09 ;{ }^{1} \mathrm{H}$ - and ${ }^{13} \mathrm{C}-\mathrm{NMR}\left(\mathrm{CD}_{3} \mathrm{OD}\right)$, see table 2.

Quercetin 3-O- $\alpha-L$-rhamnopyranosyl $(1 \rightarrow 6)-\beta$-Dglucopyranoside (4): Yellow amorphous powder; ESI-MS $m / z: 611[\mathrm{M}+\mathrm{H}]^{+} ; \mathrm{C}_{27} \mathrm{H}_{30} \mathrm{O}_{16}, \mathrm{M}=610.15 ;{ }^{1} \mathrm{H}-$ and ${ }^{13} \mathrm{C}$-NMR $\left(\mathrm{CD}_{3} \mathrm{OD}\right)$, see table 2 .

\section{RESULTS AND DISCUSSION}

Compound 1 was obtained as a white powder. The ${ }^{1}$ H-NMR spectrum of $\mathbf{1}$ showed signals of two olefinic protons at $\delta_{\mathrm{H}} 4.67(1 \mathrm{H}, \mathrm{s})$ and $4.68(1 \mathrm{H}, \mathrm{s})$, four tertiary methyls at $\delta_{\mathrm{H}} 1.22,1.12,1.07$, and 1.00 (each s, 3H); three secondary methyls at $\delta_{\mathrm{H}} 1.03$ $(3 \mathrm{H}, \mathrm{d}, J=6.8 \mathrm{~Hz}), 1.02(3 \mathrm{H}, \mathrm{d}, J=6.8 \mathrm{~Hz})$, and $0.95(3 \mathrm{H}, \mathrm{d}, J=6.4 \mathrm{~Hz})$; and four hydroxymethine protons at $\delta_{\mathrm{H}} 4.32(\mathrm{~m}), 3.97(1 \mathrm{H}, \mathrm{br} \mathrm{s}), 3.41(1 \mathrm{H}, \mathrm{m})$, and 3.14 (br d, 8.4). The two high-field doublets observed at $\delta_{\mathrm{H}} 1.14(1 \mathrm{H}, \mathrm{d}, J=3.5 \mathrm{~Hz})$ and 0.41 $(1 \mathrm{H}, \mathrm{d}, J=3.5 \mathrm{~Hz})$ are characteristic of the two protons of cyclopropane ring. The ${ }^{13} \mathrm{C}-\mathrm{NMR}$ and DEPT spectra showed the signal of 31 carbons, consisting of seven methyls, nine methylenes, nine methines, and six quaternary carbons. The analysis of NMR data suggested the structure of $\mathbf{1}$ as a cycloartane-type triterpene [5]. The HMBC correlations between $\mathrm{H}-24\left(\delta_{\mathrm{H}} 4.67\right.$ and 4.68$)$ and C-

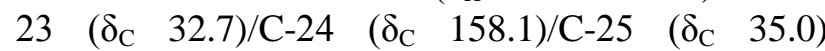
suggested an exocyclic olefinic methylene at $\mathrm{C}-24$. $\mathrm{H}-29\left(\delta_{\mathrm{H}} 1.07\right)$ and $\mathrm{H}-30\left(\delta_{\mathrm{H}} 1.12\right)$ had HMBC correlations with C-3 $\left(\delta_{\mathrm{C}} 79.8\right) / \mathrm{C}-4\left(\delta_{\mathrm{C}} 41.9\right) / \mathrm{C}-5\left(\delta_{\mathrm{C}}\right.$ $50.4)$, indicating the location of two methyl groups at C-4. The HMBC correlations between $\mathrm{H}-18\left(\delta_{\mathrm{H}}\right.$ $1.22)$ and $\mathrm{C}-12\left(\delta_{\mathrm{C}} 34.0\right) / \mathrm{C}-13\left(\delta_{\mathrm{C}} 46.9\right) / \mathrm{C}-14\left(\delta_{\mathrm{C}}\right.$ $47.3) / \mathrm{C}-17\left(\delta_{\mathrm{C}} 57.0\right) ; \mathrm{H}-28\left(\delta_{\mathrm{H}} 1.00\right)$ and $\mathrm{C}-8\left(\delta_{\mathrm{C}}\right.$ $47.7) / \mathrm{C}-13\left(\delta_{\mathrm{C}} 46.9\right) / \mathrm{C}-14\left(\delta_{\mathrm{C}} 47.3\right) / \mathrm{C}-15\left(\delta_{\mathrm{C}} 52.4\right)$ confirmed two methyl groups at $\mathrm{C}-13$ and $\mathrm{C}-14$. H$21\left(\delta_{\mathrm{H}} 0.95\right)$ was observed to have HMBC correlations with $\mathrm{C}-17\left(\delta_{\mathrm{C}} 57.0\right) / \mathrm{C}-20\left(\delta_{\mathrm{C}} 31.5\right) / \mathrm{C}-22$ $\left(\delta_{\mathrm{C}} 36.4\right)$, indicating the location of methyl group at $\mathrm{C}-20$. In addition, the HMBC correlations between $\mathrm{H}$ $26\left(\delta_{\mathrm{H}} 1.01\right), \mathrm{H}-27\left(\delta_{\mathrm{H}} 1.02\right)$ and $\mathrm{C}-24\left(\delta_{\mathrm{C}} 158.1\right) / \mathrm{C}-25$ $\left(\delta_{\mathrm{C}} 35.0\right)$ confirmed two secondary methyl groups at $\mathrm{C}$ -
25. The HBMC correlations between $\mathrm{H}-19\left(\delta_{\mathrm{H}} 1.14\right.$, $0.41)$ and carbons $\mathrm{C}-1\left(\delta_{\mathrm{C}} 34.0\right) / \mathrm{C}-5\left(\delta_{\mathrm{C}} 50.4\right) / \mathrm{C}-8$ $\left(\delta_{\mathrm{C}} 47.7\right) / \mathrm{C}-9\left(\delta_{\mathrm{C}} 20.0\right) / \mathrm{C}-10\left(\delta_{\mathrm{C}} 25.7\right)$ suggested the cyclopropane ring forming at C-9/C-10. Moreover, the COSY correlations between $\mathrm{H}-2\left(\delta_{\mathrm{H}} 1.65\right) / \mathrm{H}-3$ $\left(\delta_{\mathrm{H}} 3.14\right)$; and $\mathrm{H}-5\left(\delta_{\mathrm{H}} 1.31\right), \mathrm{H}-6\left(\delta_{\mathrm{H}} 3.97\right), \mathrm{H}-7\left(\delta_{\mathrm{H}}\right.$ $3.41)$, and $\mathrm{H}-8\left(\delta_{\mathrm{H}} 2.03\right)$; and $\mathrm{H}-15\left(\delta_{\mathrm{H}} 1.59\right.$ and $2.30) / \mathrm{H}-16 \quad\left(\delta_{\mathrm{H}} 4.02\right)$ as well as the $\mathrm{HMBC}$ correlations between $\mathrm{H}-3\left(\delta_{\mathrm{H}} 3.14\right) / \mathrm{C}-4\left(\delta_{\mathrm{C}} 41.9\right)$ and C-29 $\left(\delta_{\mathrm{C}} 25.1\right), \mathrm{H}-6\left(\delta_{\mathrm{H}} 3.97\right) / \mathrm{C}-5\left(\delta_{\mathrm{C}} 50.4\right)$ and C-7 $\left(\delta_{\mathrm{C}} 74.7\right), \mathrm{H}-7\left(\delta_{\mathrm{H}} 3.41\right) / \mathrm{C}-6\left(\delta_{\mathrm{C}} 73.1\right)$ and $\mathrm{C}-8\left(\delta_{\mathrm{C}}\right.$ $47.7)$, and $\mathrm{H}-16\left(\delta_{\mathrm{H}} 4.32\right) / \mathrm{C}-15\left(\delta_{\mathrm{C}} 52.4\right)$ and $\mathrm{C}-17$ $\left(\delta_{\mathrm{C}}\right.$ 57.0) confirmed the location of four hydroxymethine groups at C-3, C-6, C-7, and C-16. The $\beta$-configurations of the hydroxyl groups at $\mathrm{C}-3$, $\mathrm{C}-6, \mathrm{C}-7$, and $\mathrm{C}-16$ were inferred from the NOE correlations between $\mathrm{H}-3\left(\delta_{\mathrm{H}} 3.14\right) / \mathrm{H}-5_{\mathrm{ax}}\left(\delta_{\mathrm{H}} 1.31\right)$ and $\mathrm{H}_{3}-29\left(\delta_{\mathrm{H}} 1.07\right), \mathrm{H}-6\left(\delta_{\mathrm{H}} 3.97\right) / \mathrm{H}-7\left(\delta_{\mathrm{H}} 3.41\right)$ and $\mathrm{H}_{3}-29\left(\delta_{\mathrm{H}} 1.07\right), \mathrm{H}-7\left(\delta_{\mathrm{H}} 3.41\right) / \mathrm{H}-6\left(\delta_{\mathrm{H}} 3.97\right)$ and $\mathrm{H}_{3}-$ $28\left(\delta_{\mathrm{H}} 1.00\right)$, and $\mathrm{H}-16\left(\delta_{\mathrm{H}} 4.02\right) / \mathrm{H}-17_{\mathrm{ax}}\left(\delta_{\mathrm{H}} 1.64\right)$ and $\mathrm{H}_{3}-28\left(\delta_{\mathrm{H}} 1.00\right)$, respectively. The ${ }^{1} \mathrm{H}$ and ${ }^{13} \mathrm{C}-$ NMR data of $\mathbf{1}$ were identical to those of 24methylenecycloartane-3 $\beta, 6 \beta, 7 \beta, 16 \beta$-tetraol [5]. The molecular formula of $\mathbf{1}, \mathrm{C}_{31} \mathrm{H}_{52} \mathrm{O}_{4}$, was agreed with a quasi-molecular ion peak at $\mathrm{m} / z 511[\mathrm{M}+\mathrm{Na}]^{+}$ in the ESI-MS.

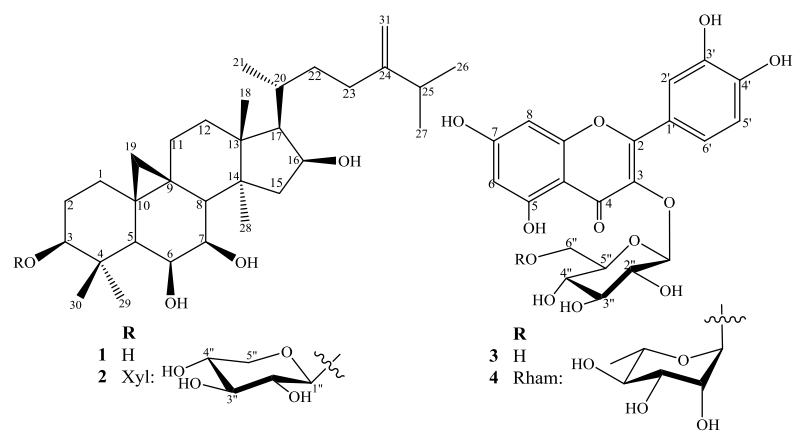

Figure 1: Chemical structures of compounds 1-4

Compound $\mathbf{2}$ was isolated as a white amorphous powder. The ${ }^{13} \mathrm{C}$-NMR spectrum of 2 revealed the signals of 36 carbons, of which five were assigned to a pentose residue and the remaining 31 to an aglycone. In addition, the ${ }^{1} \mathrm{H}$ and ${ }^{13} \mathrm{C}$-NMR spectra of $\mathbf{2}$ were almost identical to those of $\mathbf{1}$, except for the additional sugar moiety, indicating that $\mathbf{1}$ and $\mathbf{2}$ have the same aglycone. The carbon signals $\delta_{\mathrm{C}}$ $106.8,74.8,77.8,70.5$, and 66.4 could further demonstrate the existence of a pentose. The sugar moiety was elucidated as D-xylopyranoside by comparison of the NMR data of $\mathbf{2}$ with those of riparsaponin [6] and found to match (table 1). The large coupling constant of protons $\mathrm{H}-1$ ' and $\mathrm{H}-2$ ', $J$ $=6.8 \mathrm{~Hz}$, indicated $\beta$-configuration. The sugar was 
shown to be attached at $\mathrm{C}-3$ aglycone by observation of HMBC correlation between the anomeric xyl H-1' $\left(\delta_{\mathrm{H}} \quad 4.83\right)$ and C-3 $\left(\delta_{\mathrm{C}}\right.$ 88.2). Consequently, the structure of $\mathbf{2}$ was established. The molecular formula of $\mathbf{2}$ was agreed with its ESI-MS analysis $\left(\mathrm{m} / \mathrm{z}: 642,[\mathrm{M}+\mathrm{Na}]^{+}\right)$.

Compounds $\mathbf{3}$ and $\mathbf{4}$ were elucidated as flavonol glycosides in which aglycone is quercetin by analysis of ${ }^{1} \mathrm{H}$ and ${ }^{13} \mathrm{C}$-NMR spectra. Compound 3 has one sugar unit whereas $\mathbf{4}$ has two sugar units. Comparison of 1D-NMR signals of $\mathbf{3}$ with those of published data in the literature is found to match (table 2) [7]. The ${ }^{1} \mathrm{H}-\mathrm{NMR}$ spectrum of 4 indicated the presence of a rhamnosyl and a glucosyl moieties with characteristic signals at $4.50(\mathrm{H}-1 \mathrm{l} " \mathrm{~s}, \mathrm{~s})$ and 1.10 (H-6"', d, $J=6.0 \mathrm{~Hz}$ ) for rhamnose and the anomeric proton of $\beta$-glucose at $5.08(\mathrm{H}-1 ", \mathrm{~d}, J=7.2 \mathrm{~Hz})$.

Table 1: NMR spectral data for $\mathbf{1}$ and $\mathbf{2}$ and reference compounds

\begin{tabular}{|c|c|c|c|c|c|c|}
\hline \multirow{2}{*}{$\mathbf{C}$} & \multicolumn{3}{|r|}{1} & \multicolumn{3}{|r|}{2} \\
\hline & $\delta_{\mathrm{C}}^{\#, \mathrm{a}}$ & $\delta_{\mathrm{C}}^{\mathrm{a}, \mathrm{b}}$ & $\delta_{\mathrm{H}}^{\mathrm{a}, \mathrm{d}}$ (mult., $J$ in $\mathrm{Hz}$ ) & $\delta_{\mathrm{C}}^{\%, \mathrm{c}}$ & $\delta_{C}^{c, b}$ & $\delta_{\mathrm{H}}^{\mathrm{c}, \mathrm{d}}$ (mult., $J$ in $\mathrm{Hz}$ ) \\
\hline 1 & 33.9 & 34.0 & $1.51(\mathrm{~m}) / 1.70(\mathrm{~m})$ & 32.6 & 32.6 & $1.60(\mathrm{~m}) / 1.82(\mathrm{~m})$ \\
\hline 2 & 31.7 & 30.9 & $1.65(\mathrm{~m})$ & 29.2 & 29.5 & $2.07(\mathrm{~m}) / 2.35(\mathrm{~m})$ \\
\hline 3 & 79.0 & 78.8 & 3.14 (br d, 8.4) & 88.5 & 88.2 & 3.49 (br d, 8.4) \\
\hline 4 & 41.9 & 41.9 & - & 40.8 & 41.2 & - \\
\hline 5 & 50.4 & 50.9 & $1.31(\mathrm{~d}, 3.0)$ & 49.0 & 49.4 & 1.31 (br d, 3.6) \\
\hline 6 & 73.0 & 73.1 & 3.97 (br s) & 71.4 & 71.8 & $4.42(\mathrm{~m})$ \\
\hline 7 & 74.1 & 74.7 & $3.41(\mathrm{~m})$ & 72.7 & 73.1 & $3.80($ br d, 5.0) \\
\hline 8 & 47.8 & 47.7 & $2.03(\mathrm{~d}, 11.6)$ & 45.9 & 46.7 & 1.51 (br s) \\
\hline 9 & 19.9 & 20.0 & - & 24.0 & 24.4 & - \\
\hline 10 & 25.7 & 25.7 & - & 18.7 & 18.9 & - \\
\hline 11 & 26.8 & 26.9 & $1.05(\mathrm{~m}) / 2.04(\mathrm{~m})$ & 25.4 & 25.6 & $1.10(\mathrm{~m}) / 2.28(\mathrm{~m})$ \\
\hline 12 & 33.9 & 33.9 & $1.14(\mathrm{~m}) / 1.62(\mathrm{~m})$ & 32.3 & 32.8 & $1.24(\mathrm{~m}) / 1.73(\mathrm{~m})$ \\
\hline 13 & 47.0 & 46.9 & - & 45.6 & 46.0 & - \\
\hline 14 & 47.1 & 47.3 & & 45.4 & 45.8 & - \\
\hline 15 & 53.3 & 52.4 & $1.59(\mathrm{~m}) / 2.30(\mathrm{~m})$ & 51.7 & 52.1 & $2.18(\mathrm{~m}) / 2.85(\mathrm{~m})$ \\
\hline 16 & 72.3 & 73.6 & $4.32(\mathrm{~m})$ & 70.7 & 71.3 & $4.70(\mathrm{~m})$ \\
\hline 17 & 57.1 & 57.0 & $1.64(\mathrm{~m})$ & 55.4 & 56.0 & $1.82(\mathrm{~m})$ \\
\hline 18 & 19.3 & 19.9 & $1.22(\mathrm{~s})$ & 19.4 & 19.5 & $1.57(\mathrm{~s})$ \\
\hline 19 & 32.9 & 33.0 & $0.41(\mathrm{~d}, 3.5) / 1.14(\mathrm{~d}, 3.5)$ & 31.4 & 31.7 & $0.53(\mathrm{~d}, 3.5) / 1.5541(\mathrm{~d}, 3.5)$ \\
\hline 20 & 31.3 & 31.5 & $1.84(\mathrm{~m})$ & 29.8 & 30.2 & $2.28(\mathrm{~m})$ \\
\hline 21 & 18.9 & 18.6 & $0.95(\mathrm{~d}, 6.4)$ & 17.9 & 17.8 & $1.12(\mathrm{~d}, 6.8)$ \\
\hline 22 & 36.1 & 36.4 & $1.17(\mathrm{~m}) / 1.85(\mathrm{~m})$ & 34.5 & 35.0 & $1.42(\mathrm{~m}) / 2.24(\mathrm{~m})$ \\
\hline 23 & 32.8 & 32.7 & $2.00(\mathrm{~m}) / 2.15(\mathrm{~m})$ & 31.4 & 32.8 & $1.50(\mathrm{~m}) / 1.74(\mathrm{~m})$ \\
\hline 24 & 157.6 & 158.1 & - & 156.2 & 156.5 & - \\
\hline 25 & 34.5 & 35.0 & $2.26(\mathrm{~m})$ & 33.1 & 33.4 & $2.32(\mathrm{~m})$ \\
\hline 26 & 22.4 & 22.4 & $1.02(\mathrm{~d}, 6.8)$ & 21.8 & 21.4 & $1.04(\mathrm{~d}, 6.8)$ \\
\hline 27 & 22.5 & 22.5 & $1.03(\mathrm{~d}, 6.8)$ & 21.9 & 21.5 & $1.06(\mathrm{~d}, 6.8)$ \\
\hline 28 & 20.6 & 20.2 & $1.00(\mathrm{~s})$ & 19.4 & 19.4 & $1.27(\mathrm{~s})$ \\
\hline 29 & 25.7 & 25.1 & $1.07(\mathrm{~s})$ & 24.1 & 24.1 & $1.55(\mathrm{~s})$ \\
\hline 30 & 16.9 & 16.0 & $1.12(\mathrm{~s})$ & 16.4 & 16.5 & $1.67(\mathrm{~s})$ \\
\hline 31 & 106.7 & 106.6 & $4.67(\mathrm{~s}) / 4.68(\mathrm{~s})$ & 105.8 & 105.7 & $4.82(\mathrm{~s}) / 4.89(\mathrm{~s})$ \\
\hline \multicolumn{7}{|c|}{ 3-O-Xyl } \\
\hline $1^{\prime}$ & & & & 105.9 & 106.8 & $4.83(\mathrm{~d}, 6.8)$ \\
\hline $2^{\prime}$ & & & & 73.8 & 74.8 & $3.99(\mathrm{~m})$ \\
\hline $3^{\prime}$ & & & & 76.8 & 77.8 & $4.11(\mathrm{t}, 8.8)$ \\
\hline $4^{\prime}$ & & & & 69.7 & 70.5 & $4.18(\mathrm{~m})$ \\
\hline $5^{\prime}$ & & & & 65.6 & 66.4 & $3.71(\mathrm{~m}) / 4.35(\mathrm{~m})$ \\
\hline
\end{tabular}

Measured in ${ }^{\text {a) }} \mathrm{CD}_{3} \mathrm{OD},{ }^{\mathrm{b})} 100 \mathrm{MHz},{ }^{\mathrm{c})}$ piridine- $\mathrm{d}_{5},{ }^{\mathrm{d})} 400 \mathrm{MHz}$.

${ }^{\#)} \delta_{\mathrm{C}}$ of 24 -methylenecycloartane- $3 \beta, 6 \beta, 7 \beta, 16 \beta$-tetraol [5], ${ }^{\% /} \delta_{\mathrm{C}}$ of riparsaponin [6]. 


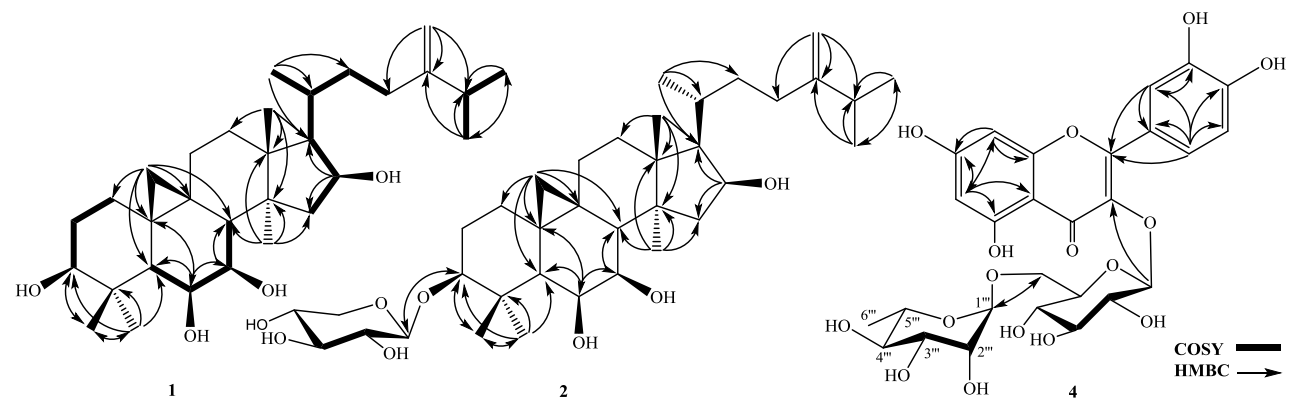

Figure 2: The key HMBC and COSY correlations of compounds 1, 2 and 4

Table 2: ${ }^{1} \mathrm{H}$ - and ${ }^{13} \mathrm{C}-\mathrm{NMR}$ data for $\mathbf{3}$ and $\mathbf{4}$ and reference compounds

\begin{tabular}{|c|c|c|c|c|c|c|}
\hline \multirow{2}{*}{$\mathbf{C}$} & \multicolumn{3}{|r|}{3} & \multicolumn{3}{|r|}{4} \\
\hline & $\delta_{C}^{\$, a}$ & $\delta_{C}^{\mathrm{a}, \mathrm{b}}$ & $\delta_{\mathrm{H}}^{\text {a) }}$ (mult., $J$ in Hz) & $\delta_{C}^{\&, a}$ & $\delta_{\mathrm{C}}^{\mathrm{a}, \mathrm{b}}$ & $\delta_{\mathrm{H}}^{\text {a) }}($ mult., $J$ in $\mathrm{Hz})$ \\
\hline 2 & 156.8 & 159.0 & - & 158.5 & 159.4 & - \\
\hline 3 & 133.8 & 135.6 & - & 135.6 & 135.6 & - \\
\hline 4 & 177.5 & 179.5 & - & 179.4 & 179.4 & - \\
\hline 5 & 161.7 & 163.0 & - & 162.9 & 162.9 & - \\
\hline 6 & 99.1 & 99.84 & $6.19(\mathrm{~s})$ & 99.9 & 99.9 & $6.18(\mathrm{~s})$ \\
\hline 7 & 164.2 & 166.0 & - & 166.0 & 166.0 & - \\
\hline 8 & 93.9 & 94.7 & $6.38(\mathrm{~s})$ & 94.9 & 94.8 & $6.36(\mathrm{~s})$ \\
\hline 9 & 156.6 & 158.5 & - & 159.3 & 159.3 & - \\
\hline 10 & 104.6 & 105.7 & - & 105.6 & 105.6 & - \\
\hline $1^{\prime}$ & 121.6 & 123.1 & - & 123.1 & 123.1 & - \\
\hline $2^{\prime}$ & 115.6 & 116.0 & $7.70(\mathrm{~d}, 2.0)$ & 116.0 & 117.7 & $7.65(\mathrm{~s})$ \\
\hline $3^{\prime}$ & 145.2 & 145.9 & - & 145.8 & 145.8 & - \\
\hline $4^{\prime}$ & 148.9 & 149.8 & - & 149.8 & 149.8 & - \\
\hline $5^{\prime}$ & 116.6 & 117.5 & $6.88(\mathrm{~d}, 8.0)$ & 117.7 & 116.0 & $6.85(\mathrm{~d}, 8.2)$ \\
\hline $6^{\prime}$ & 120.0 & 123.2 & $7.57(\mathrm{dd}, 2.0,8.0)$ & 123.5 & 123.5 & $7.57(\mathrm{~d}, 8.2)$ \\
\hline $1 "$ & 101.3 & 104.3 & $5.25(\mathrm{~d}, 7.2)$ & 104.7 & 104.7 & $5.08(\mathrm{~d}, 7.2)$ \\
\hline $2 "$ & 74.5 & 75.7 & $3.47(\mathrm{dd}, 7.2,8.2)$ & 75.7 & 75.8 & $3.42(\mathrm{~m})$ \\
\hline $3^{\prime \prime}$ & 76.9 & 78.1 & $3.41(\mathrm{t}, 8.2)$ & 78.2 & 78.2 & $3.40(\mathrm{~m})$ \\
\hline 4" & 70.4 & 71.2 & $3.21(\mathrm{~m})$ & 71.3 & 71.4 & $3.25(\mathrm{~m})$ \\
\hline $5 "$ & 78.0 & 78.4 & $3.32(\mathrm{~m})$ & 77.1 & 77.2 & $3.30(\mathrm{~m})$ \\
\hline \multirow[t]{2}{*}{$6^{\prime \prime}$} & 61.4 & 62.5 & $3.55(\mathrm{dd}, 5.6,11.4)$ & 68.5 & 68.5 & $3.40(\mathrm{dd}, 5.6,11.4)$ \\
\hline & & & $3.70(\mathrm{dd}, 2.4,11.4)$ & & & $3.78(\mathrm{dd}, 2.4,11.4)$ \\
\hline 1"' & & & & 102.4 & 102.4 & 4.50 (br s) \\
\hline $2^{\prime \prime \prime}$ & & & & 72.0 & 72.1 & $3.62(\mathrm{~m})$ \\
\hline $3^{\prime \prime \prime}$ & & & & 72.2 & 72.2 & $3.52(\mathrm{~m})$ \\
\hline $4 " '$ & & & & 73.9 & 73.9 & $3.25(\mathrm{~m})$ \\
\hline $5 " '$ & & & & 69.7 & 69.7 & $3.41(\mathrm{~m})$ \\
\hline 6"' & & & & 17.9 & 17.9 & $1.10(\mathrm{~d}, 6.0)$ \\
\hline
\end{tabular}

Measured in a) $\mathrm{CD}_{3} \mathrm{OD},{ }^{\text {b) }} 100 \mathrm{MHz},{ }^{\mathrm{c})} 400 \mathrm{MHz},{ }^{\$} \delta_{\mathrm{C}}$ of quercetin-3-O- $\beta$-glucopyranoside [7], ${ }^{\&)} \delta_{\mathrm{C}}$ of quercetin 3 -O- $\alpha$-Lrhamnopyranosyl $(1 \rightarrow 6) \beta$-D-glucopyranoside [8].

The HMBC correlations between H-6" and C-1"', H$1 " '$ and C-6", and $\mathrm{H}-1$ " and C-3 $\left(\delta_{\mathrm{C}} 135.6\right)$ confirmed the linkage $(1 \rightarrow 6)$ between rhamnosyl and glucosyl moieties and the glucopyranosyl moiety connected with aglycone quercertin at $\mathrm{C}-3$. Moreover, the ${ }^{1} \mathrm{H}$ and ${ }^{13} \mathrm{C}$-NMR data of $\mathbf{4}$ were identical with those reported in the literature (table 2) [8]. Consequently, the structure of $\mathbf{3}$ and $\mathbf{4}$ was elucidated as quercetin-
$3-O-\beta$-D-glucopyranoside, and quercetin $3-O-\alpha-\mathrm{L}-$ rhamnopyranosyl $\quad(1 \rightarrow 6) \quad$ glucopyranoside, respectively.

Acknowledgement. This research was supported by University of Science, VNU under grant number TN.16.11. 


\section{REFERENCES}

1. V. V. Chi. The Dictionary of Medicinal Plants in Vietnam, Medical Publising House, 574-575 (2012).

2. U. C. Bapat, D. R. Mhapsekar. Phytochemical investigations and antimicrobial and anticancer activities of Homonoia riparia Lour, Journal of Pharmacy and Pharmaceutical Sciences, 237-243 (2014).

3. J. -H. Yu, Y. Shen, H.-B. Liu, Y. Leng, H. Zhang, J.M. Yue. Dammarane-type triterpenoids as $11 \beta$ HSD1 inhibitors from Homonoia riparia, Organic \& Biomolecular Chemistry, 12, 4716-4722 (2014).

4. S. -M. Yang, X. -K. Liu, C. Qing, D. -G. Wu, D. -Y. Zhu. Chemical constituents from the roots of Homonoia riparia, Yao Xue Xue Bao, 42, 292-296 (2007).

\section{Corresponding author: Phan Van Kiem}

Institute of Marine Biochemistry

Vietnam Academy of Science and Technology

No. 18, Hoang Quoc Viet Road, Cau Giay Dist., Hanoi

E-mail:phankiem@yahoo.com.
5. I. Lee, J. Kim, Y. S. Kim, N. H. Yoo, C.-S. Kim, K. Jo, J.-H. Kim, T. T. Bach, J. S. Kim. Cycloartanetype triterpenes from the leaves of Homonoia riparia with VEGF-induced angiogenesis inhibitory activity, Journal of Natural Products, 75, 1312-1318 (2012).

6. F. Xu, X. Zhao, L. Yang, X. Wang, J. Zhao. A new cycloartane-type triterpenoid saponin xanthine oxidase inhibitor from Homonoia riparia Lour, Molecules, 19, 13422-13431 (2014).

7. H. -Y. Lin, Y. -H. Kuo, Y. -L. Lin, W. Chiang. Antioxidative effect and active components from leaves of Lotus (Nelumbo nucifera), Journal of Agricultural and Food Chemistry, 57, 6623-6629 (2009).

8. M. -A. Beck, H. Häberlein. Flavonol glycosides from Eschscholtzia californica, Phytochemistry, 50, 329332 (1999). 